\title{
New aspects in the study of carbon-hydrogen interaction in hydrogenated carbon nanotubes for energy storage applications
}

\author{
M. Brzhezinskayaa, ${ }^{a}$, E.A. Belenkov ${ }^{\mathrm{b}}$, V.A. Greshnyakov ${ }^{\mathrm{b}}$, G.E. Yalovega ${ }^{\mathrm{c}}$, I.O. Bashkin ${ }^{\mathrm{d}}$
}

\author{
${ }^{a}$ Helmholtz-Zentrum Berlin für Materialien und Energie, Albert-Einstein-Str. 15, 12489 Berlin, \\ Germany \\ ${ }^{\mathrm{b}}$ Chelyabinsk State University, Bratiev Kashirinykh Ave. 129, 454001 Chelyabinsk, Russia \\ ${ }^{c}$ Southern Federal University, Sorge Str. 5, 344090 Rostov-on-Don, Russia \\ ${ }^{\mathrm{d}}$ Institute of Solid State Physics RAS, Akademika Osipyana 2, 142432 Chernogolovka, Russia \\ * Corresponding author: \\ Dr. habil. Maria Brzhezinskaya \\ Helmholtz-Zentrum Berlin für Materialien und Energie, \\ Albert-Einstein-Str. 15, 12489 Berlin, Germany \\ E-mail: maria.brzhezinskaya@helmholtz-berlin.de
}

\begin{abstract}
The paper presents the results of the combined investigation of changes in the carbon nanotubes structure caused by hydrogenation. Hydrogen adsorption by bundles of single-wall carbon nanotubes (SWNTs) was simulated by the molecular mechanics and DFT-LDA methods and applied to interpret the results of the experimental study carried out by using high-resolution X-ray absorption and photoelectron spectroscopy. As a result, new aspects of physical and chemical adsorption of hydrogen atoms on SWNTs were revealed, as well as preferable ways for SWNTs hydrogenation. Due to van-der-Waals interactions, location of hydrogen molecules inside the channels is energetically more favorable than that outside the channels. Chemical covalent bonds between hydrogen atoms and nanotube walls arise with formation of the $\mathrm{C}_{4} \mathrm{H}$ and $\mathrm{C}_{2} \mathrm{H}$ phases. However, not the entire surface of the SWNTs located inside the bundles is accessible for hydrogenation but only segments adjacent to triangular pores.
\end{abstract}

Keywords: hydrogenated single-wall carbon nanotubes, X-ray absorption spectroscopy, X-ray photoelectron spectroscopy, density functional calculations, finite difference method, energy storage materials

Declarations of interest: none. 


\section{Introduction}

During the last decades, the world-wide power consumption has increased almost 15fold. The most actively exploited primary sources of power are petroleum products (30\%), coal (20\%), natural gas (20\%) and nuclear fuel (6\%) [1]. Due to this, carbon dioxide releases into the atmosphere have grown 4.5 times during the last 50 years, which lays the groundwork for considerable ecological problems. At the same time, estimates of the conventional energy source resources showed that the oil and gas resources are sufficient for no more than 100 years. Thus, one of the basic problems of modern power industry is to find alternative renewable power sources and efficiently increase their share in the general power consumption. Hydrogen offers an alternative fuel source in the event of a petroleum shortage [2]. However, none of the current methods for hydrogen storage (at high pressure, in the absorbed state at low temperatures, in the liquid state, in the form of metal hydrides or intermetallics) do not ensure so safe reversible storage and compact transportation of gaseous hydrogen as carbon nanotubes (CNTs) [3-13]. Therefore, a lot of investigations are now aimed at studying peculiar features of hydrogen sorption by CNTs that exhibit extremely high porosity.

At the same time, the experimental and theoretical results are at once encouraging, misleading, and fragmentary. This is first due to the lack of a universal technique for carbon nanotube hydrogenation, e.g., physical or chemical hydrogenation. Another reason is the gap between experimental and theoretical studies despite significant achievements in simulation of interaction of atomic and molecular hydrogen with CNTs (e.g. [14-16]). In addition, chemical reagents and reaction conditions may be different even in hydrogenation of one and the same type. For instance, in work [17] single-wall carbon nanotubes (SWNTs) were hydrogenated using polyamine reagents, while in other studies hydrogenation was accomplished based on reaction with molecular hydrogen [18] or in liquid ammonia [19]. The processes of nanotube hydrogenation may differ also in nanotube types (e.g., MWNTs [20] and SWNTs [18, 21, 22]), procedures of CNT fabrication (e.g., Hipco [23], chemical vapor deposition (CVD) [18] or arcdischarge [19]) and preparation (e.g., powder, bucky paper), and, in addition, in the hydrogen form (atomic or ionic) $[18,24]$ used for the treatment and usage of pretreatment for creating extra defects in graphene layers forming CNTs [24]. Additional difficulties arise in attempts to consolidate all the results due the fact that hydrogenated CNTs (H-CNTs) investigated in various studies have different hydrogen concentration, which, as shown earlier for fluorinated multi-wall carbon nanotubes, can essentially affect the variations in the H-CNT structure [25].

From the standpoint of further application of CNTs for hydrogen storage, the investigation addressing the possibility of multiple reversible hydrogenations and dehydrogenations of carbon nanotubes is the most critical. To this end, detailed understanding 
of CNT structure changes caused by hydrogenation and studying local structure of the carbonhydrogen $(\mathrm{C}-\mathrm{H})$ bonding sites are of the utmost importance. Therefore, we attempted to eliminate this gap by interpreting the results of the experimental study by simulating physical adsorption of hydrogen by bundles of the SWNTs using the molecular dynamics and DFT-LDA methods. The CNTs models obtained as a result of such simulation were used to theoretically analyze the absorption spectra. The experimental data were obtained by high-resolution nearedge X-ray absorption fine structure (NEXAFS) spectroscopy and X-ray photoelectron spectroscopy (XPS). As a result, new aspects of the nature of interaction between hydrogen atoms and carbon atoms of SWNTs were revealed, as well as preferable ways for SWNTs hydrogenation.

\section{Material and methods}

The SWNTs were synthesized by the arc discharge evaporation method. Purification resulted in a $\sim 98$ wt.\% content of SWNTs. The SWNT average diameter was $1.5 \mathrm{~nm}(1.4-1.6$ $\mathrm{nm})$. The final material consisted of large $(\sim 100 \mu \mathrm{m})$ nanotube mats [26]. The hydrogenation of the SWNTs was performed by treating them at high hydrogen pressure P 5.0 GPa and elevated temperature $T_{H} \sim 500^{\circ} \mathrm{C}$ [27]. The obtained sample of $\mathrm{H}-\mathrm{SWNT}$ contained $5.335 \mathrm{wt} \%$ of hydrogen. The content of hydrogen in the H-SWNTs was determined by combustion method. The more detailed description of the hydrogenation procedure is given in [28].

The X-ray absorption and photoelectron spectra were measured at the Russian-German beamline of the BESSY II storage ring facility at Helmholtz-Zentrum Berlin (Berlin, Germany) [29]. Powders of the SWNTs and H-SWNTs were rubbed into the scratched surface of a pure substrate. The NEXAFS spectra were obtained by recording the total electron yield (TEY) of the $\mathrm{X}$-ray photoemission in the mode of the drain current measurement. The X-ray absorption and photoelectron spectra were measured in ultrahigh vacuum $\left(\sim 2 \times 10^{-10}\right.$ Torr $)$. The samples were mounted at the angle of $\sim 45^{\circ}$ to the incident beam. The size of the focused spot on the sample was about $0.2 \times 0.1 \mathrm{~mm}$.

The monochromator energy resolution $\Delta E$ in the vicinity of the $\mathrm{C} 1 s \mathrm{X}$-ray absorption edge (photon energy $h v \sim 285 \mathrm{eV}$ ) was $\sim 70 \mathrm{meV}$. The X-ray absorption spectra were normalized to the incident photon flux. The photon energy in the range of the fine structure of the $\mathrm{C} 1 s \mathrm{X}$-ray absorption spectrum was calibrated against the energy position of the first narrow peak in the $\mathrm{C}$ $K$-edge spectrum of highly oriented pyrolytic graphite (HOPG, $285.45 \mathrm{eV}$ [25]).

The X-ray photoemission spectra were acquired using the Phoibos 150 electron energy analyzer (Specs). The C $1 s$ spectra were acquired at photon energies $(h v)$ of $485-1030 \mathrm{eV}$ and analyzer pass energies $(P E)$ of $2-10 \mathrm{eV}$ with the overall (monochromator and analyzer) energy 
resolution of $\sim 100-200 \mathrm{meV}$. The $\mathrm{C} 1 s$, Au $4 f$ and valence band spectra of the reference samples ( $\mathrm{HOPG}$ and $\mathrm{Au}$ ) were measured to calibrate the analyzer work function. The detection angle was close to that of normal emission. To analyze the data, the spectra were fitted by the Gaussian/Lorentzian convolution functions with simultaneous optimization of the background parameters by using the UNIFIT software [30]. The C $1 s$ line asymmetry was described with the Doniach-Šunjić (DS) function. The background was modelled by the equation $\mathrm{U}(\mathrm{E})=\mathrm{a}+\mathrm{s} \cdot \mathrm{S}(\mathrm{E})+\mathrm{t} \cdot \mathrm{T}(\mathrm{E})$, where $S(E)$ is the Shirley background and $T(E)$ is the Tougaard background.

Physical adsorption of hydrogen in the nanotube bundles was simulated by the molecularmechanics method (MM2) [31]. The calculation was performed for a cluster consisting of seven closely-packed fragments of the $(10,10)$ SWNTs. The number of carbon atoms in each SWNT fragment was 400. Hydrogen molecule $\mathrm{H}_{2}$ was located in the bundle central part between SWNTs. After that, the model was geometrically optimized, due to which the hydrogen molecule position corresponding to the minimum total energy was found. The hydrogen chemical adsorption was calculated in the Quantum ESPRESSO software package [32] by the density functional theory (DFT) method in the local density approximation (LDA-PZ) [33]. For calculation, the norm-conserving Troullier-Martins pseudopotential was used. Calculation was performed by using the Brillouin-zone $k$-point grids $8 \times 8 \times 8$. Wave functions were expanded in a truncated basis set of plane waves. The cut-off energy was 60 Rydberg. Criteria for stopping geometric optimization of the structure were the atomic force values less than $1 \mathrm{meV} / \AA$ and stress values less $1.3 \mathrm{meV} / \AA^{3}$.

Theoretical C $K$-edge NEXAFS spectra were obtained using the non-muffin-tin approach with the finite difference method (FDMNES2012) for solving the Schrödinger equation [34].

\section{Results and discussion}

The high-resolution photoemission spectrum of pristine SWNTs is shown in Fig. 1 in comparison with the spectrum obtained for HOPG (Fig. 1, Inset). The experimental conditions ( $h v=485 \mathrm{eV}, P E=5 \mathrm{eV}$ ) provided both high energy resolution and optimal bulk sensitivity of $\sim 0.6$ $\mathrm{nm}$. For HOPG, the $\mathrm{C} 1 s$ spectral lineshape is described by the parameters (binding energy $B E=284.49 \mathrm{eV}, G=0.19 \mathrm{eV}, L=0.16 \mathrm{eV}, \alpha=0.1)$ which are in agreement with the data reported earlier [35]. The high-resolution C $1 s$ spectrum of pristine SWNTs (component $A, \mathrm{C}-\mathrm{C} s p^{2}$ ) is broader than that for HOPG $(B E=284.59 \mathrm{eV}, G=0.30 \mathrm{eV}, L=0.16 \mathrm{eV}, \alpha=0.1)$. A small upshift in $B E \sim 0.1 \mathrm{eV}$ is observed. The low-intensity component at $B E=286.19 \mathrm{eV}\left(\mathrm{C}-\mathrm{O}_{\mathrm{x}}\right)$ may be related to oxidation. The broadening effect can be attributed to slightly different BEs for SWNTs with diameters varying between 1.4 and $1.6 \mathrm{~nm}$ or to the presence of a small amount of defects in the 
SWNT structure. As we reported earlier [36], the small upshift of the C 1 s peak for SWNTs can be attributed to the Fermi level upshift and corresponding change in the work function. The main peak in the SWNTs C $1 s$ photoelectron spectrum is accompanied also by a satellite whose position with respect to the $\mathrm{C} 1 \mathrm{~s}$ peak is $6.3 \mathrm{eV}(B E=290.89 \mathrm{eV})$. The satellite is associated with discrete energy losses of emitted C $1 s$ photoelectrons for valence electron excitation in the graphene layer (shake up, inelastic scattering, and plasmon excitations) [37]. The energy gap between the satellite and main $\mathrm{C} 1 s$ line is close to the $\pi$ plasmon energy in the process of characteristic electron energy losses in CNTs [38].

The H-SWNT C $1 s$ spectra have a multicomponent structure (Fig. 2 and Fig. 3). To control the sample composition, survey photoelectron spectra at $h v=1030 \mathrm{eV}$ were also measured. They did not show the presence of oxygen in the H-SWNT samples (see Fig. 2, Inset). The C $1 s$ line of H-SWNTs consists of five components colored differently for different H/C ratios. Component $A$ at $B E=284.59 \mathrm{eV}(G=0.30 \mathrm{eV}, L=0.16 \mathrm{eV}, \alpha=0.1)$ can be assigned to carbon atoms bound to the other carbon atoms, which corresponds to the spectrum of pristine SWNTs. As a result of the hydrogenation, four additional peaks emerged. They are distanced from the CC peak by $0.1 \mathrm{eV}, 0.6 \mathrm{eV}, 2.2 \mathrm{eV}$ and $4.9 \mathrm{eV}$, respectively. The binding energy of the first of these four peaks $\left(A^{\prime}\right)$ is lower than that of the main C-C peak $(A)$. As it was shown in [39] for hydrogenated graphene, peak $A^{\prime}$ may be assigned to carbon atoms next to the $\mathrm{C}-\mathrm{H}$ bonds and correspond to formation of the $\mathrm{C}_{4} \mathrm{H}$ phase. Note that the presence of this feature in the H-SWNTs spectrum has also been shown in this study for the first time, though the assumption on the existing overlapping of electronic density between carbon atoms bound and unbound to hydrogen atoms resulting from SWNT hydrogenation was made in our previous work in analyzing calculated angular-depended C $1 s$ NEXAFS spectra of H-SWNTs [40].

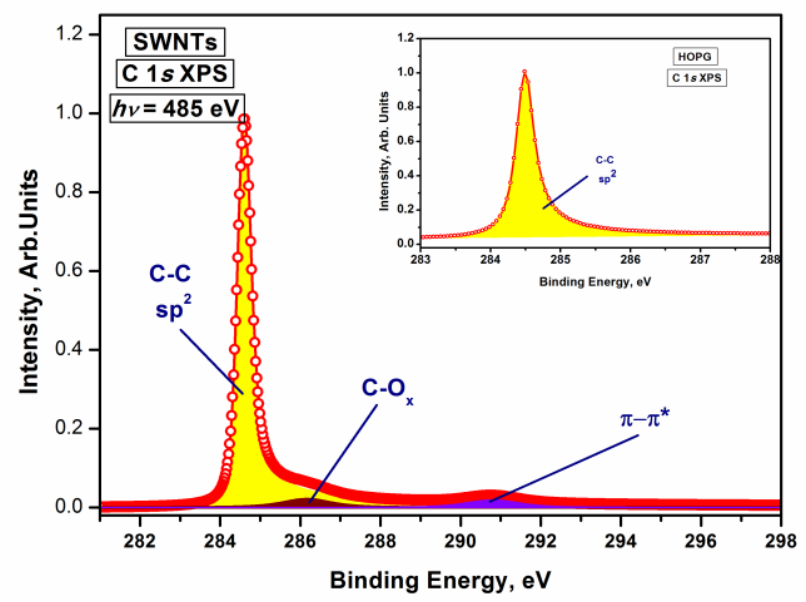

Fig. 1. The C $1 s$ photoelectron spectra of SWNT and HOPG (inset). 
Peaks with $B E=285.18 \mathrm{eV}\left(A_{1}\right), 286.77 \mathrm{eV}(B)$ and $289.44 \mathrm{eV}(C)$ correspond to the contribution of carbon atoms participating in formation of chemical bonds between the carbon and hydrogen atoms. The mentioned interaction between $\mathrm{C}$ and $\mathrm{H}$ atoms results in formation of carbon-hydrogen phases. Hydrogen has higher electronegativity than carbon. As a result, charge transfer from carbon atoms to hydrogen atoms arose. This pronounced difference in positions of the $A_{1}, B$ and $C$ peaks $\left(\Delta_{A 1-B}=1.59 \mathrm{eV}\right.$ and $\left.\Delta_{B-C}=2.67 \mathrm{eV}\right)$ reflects differences in the natures of interactions between carbon and hydrogen atoms in the observed $\mathrm{C}-\mathrm{H}$ phases formed in $\mathrm{H}$ SWNTs. Phase $1(B E=286.77 \mathrm{eV})$ is characterized by the most intense electron-density transfer from carbon atoms to hydrogen atoms. This fact confirmed the results of our simulation (see below) that a part of hydrogen atoms bound covalently to the SWNTs graphene layers $\left(\mathrm{C}_{2} \mathrm{H}\right.$ phase).

The chemical shift in the positions of $\mathrm{C} 1 \mathrm{~s}$ peak for phase 2 is much smaller for than that for phase $1(2.2 \mathrm{eV})$. Therefore, we can conclude that hydrocarbon phase 2 exhibits a significantly weaker electron-density transfer from carbon atoms to hydrogen atoms, and, hence, phase 2 has a weaker chemical bonding between $\mathrm{C}$ and $\mathrm{H}$ atoms. In that way, the distances between carbon and hydrogen atoms in phase $1\left((1,2)\right.$ phase $\left.\left(\mathrm{C}_{2} \mathrm{H}\right)\right)$ are shorter as compared to that of phase $2\left((1,4)\right.$ phase $\left.\mathrm{C}_{4} \mathrm{H}\right)$. At the same time, we should note that it is impossible to resolve in the H-SWNTs spectrum the contributions of $s p^{3}$ carbon at $B E \sim 285.5 \mathrm{eV}$ within the interpretation suggested in [41] that an origin of $s p^{3}$ carbon $(B E \sim 285.5 \mathrm{eV})$ of $\mathrm{C} 1 s$ XPS spectra for carbon materials is charging effect, which is caused by the disconnection of the pathway of electrons between $s p^{2}$ carbon and $s p^{3}$ carbon.

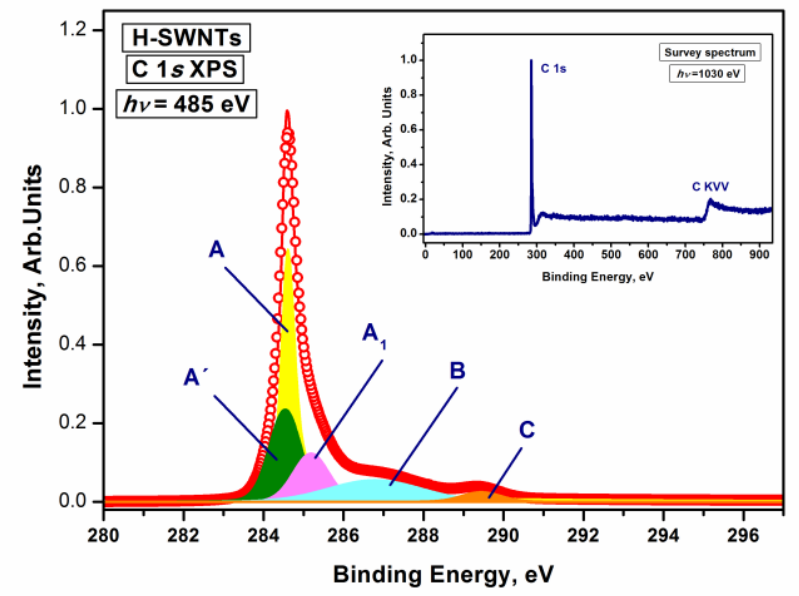

Fig. 2. C 1s photoelectron and survey (inset) spectra of H-SWNT.

It is important to compare the C $1 s$ photoelectron spectra recorded at $h v=485 \mathrm{eV}$ and $h v=1030 \mathrm{eV}$ (Fig. 3). With $h v$ increasing up to $1030 \mathrm{eV}$, relative intensities of peaks $A_{l}$ and $B$ 
(contribution of $\mathrm{C}-\mathrm{H}$ phases) increased, while relative intensities of peaks $A$ and $A^{\prime}$ decreased. It is reasonable to relate these changes to the increase in the probing depth (from 0.6 to $2 \mathrm{~nm}$ ). This experimental fact is confirmed by our theoretical conclusion (see below) that location of hydrogen molecules inside the channels is energetically more favorable than that outside the channels.

Peaks $A_{1}, B$ and $C$ are critical for the problem of determining the hydrogen atom content in the samples by using simple formula $\eta(\%)=\left[\left(S_{A 1}+S_{B}+S_{C}\right) /\left(S_{A^{\prime}}+S_{A}+S_{A 1}+S_{B}+S_{C}\right)\right] \cdot 100 \%$, where $S_{A^{\prime}}$, $S_{A}, S_{A 1}, S_{B}$ and $S_{C}$ represent the areas under the peaks in the C $1 s$ photoelectron spectra. According to this formula, atomic concentration of hydrogen in investigated H-SWNTs is $32 \%$. Using the same formula, we can see that the atomic concentration of hydrogen in the investigated H-SWNTs at the depth of $2 \mathrm{~nm}$ is $54 \%$, which agrees well with the result obtained in [21]. It is worth noting that in this case the peak $B$ relative area changes more than twice (from $15 \%$ to $37 \%$ ). Thus, this is one more confirmation of the fact that just peak $B$ represents the contribution of carbon atoms covalently bound with hydrogen atoms. The very contribution of this $\mathrm{C}$-H phase dominates in the $\mathrm{C} 1 s$ absorption spectrum (Fig. 4). At the same time, the $C$-peak relative area shows that the number of destroyed SWNTs does not exceed 3\%.

In the C $\mathrm{K}$-edge spectrum of pristine SWNTs (Fig. 4(a)), the most pronounced features $A-a$ and $B-F$ reflect transitions of the $\mathrm{C} 1 s$ electrons into free states of the conductivity band formed from the C $2 p_{z}$ and C $2 p_{x, y}$ electron states with the $\pi^{*}$ and $\sigma^{*}$ symmetry, respectively [42]. The C $1 s$ spectra of SWNTs and H-SWNTs differ significantly. A drastic decrease in the $A$ peak intensity and emergence of new bands $B_{1}^{*}(288.8 \mathrm{eV}), B_{2}^{*}(287.3 \mathrm{eV}), B^{*}, C^{*}, D^{*}$ and $E^{*}-$ $F^{*}$ are observed (Fig. 4(a)). It is important to pay attention that band $A$ typical of the SWNT spectrum is still observed. This observation indicates incompleteness of SWNT hydrogenation which is also confirmed by the X-ray photoelectron spectroscopy data given above.

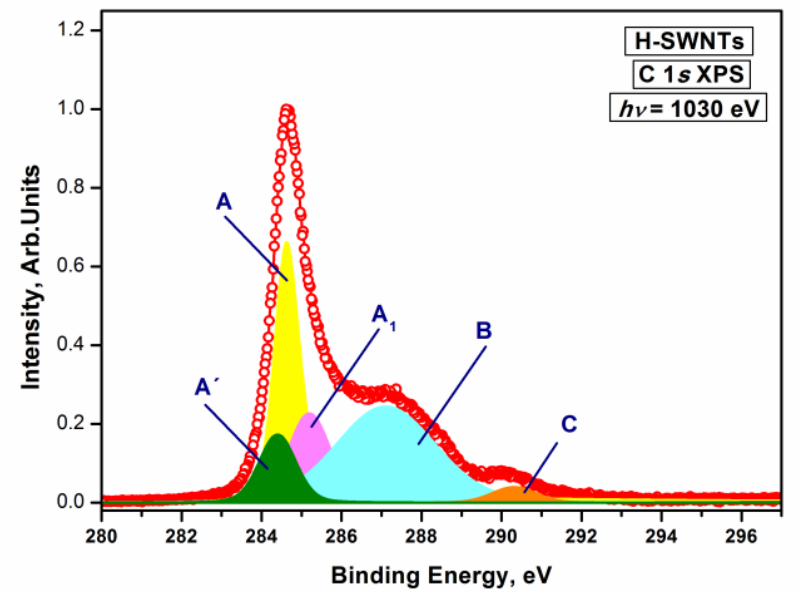

Fig. 3. C 1s photoelectron spectrum of H-SWNT, measured at $h v=1030 \mathrm{eV}$. 
It would be natural to interpret the changes in the H-SWNT C $1 s$ spectra as a result of chemical interaction between the hydrogen atoms and carbon atoms of graphene layers forming the SWNT walls. The most pronounced changes are reduction of the $\pi$ - (peak $A$ ) and $\sigma$ - (peak $B$ ) resonances and arising of new absorption bands in the 286-292 eV and 303-310 eV energy ranges. As we have shown earlier in analyzing theoretical $\mathrm{C} 1 s$ absorption spectra, chemical bonding of $\mathrm{H}$ atoms with $\mathrm{C}$ atoms takes place mainly occurs as bonding of the hydrogen atoms to carbon atoms on SWNTs walls with formation of $\sigma(\mathrm{C}-\mathrm{H})$ bonds due to covalent mixing of $\mathrm{H} 1 s$ - and $\mathrm{C} 2 p_{z}$-states [40]. This $\mathrm{C}-\mathrm{H}$ bonds formation modifies the $\mathrm{C}$ atoms configuration from triangular in pristine SWNTs to nearly tetrahedral in H-SWNTs; this is possible only when the $s p^{2}$ hybridization of $\mathrm{C}$ atom valence states changes to the $s p^{3}$ hybridization. At the same time, the arisen feature $B_{2} *(287.3 \mathrm{eV})$ reflects the $\mathrm{C}-\mathrm{H}$ bonding well-known for hydrocarbons [42]. The new peaks $B_{1} *$ and $B_{2} *$ in the $\mathrm{C} K$-edge spectrum of H-SWNTs could arise as a result of two different ways in which $\mathrm{H}$ atoms are connected to the SWNT graphene layers with forming $\sigma(\mathrm{C}-$ $\mathrm{H})$ bonds with diverse values of charge transfer from carbon to hydrogen atoms. According to our theoretical investigation, peak $B_{1} *$ reflects the contribution of $\mathrm{C}$ atoms unbound with $\mathrm{H}$ atoms. These $\mathrm{C}$ atoms are located between two $\mathrm{C}$ atoms bound to the $\mathrm{H}$ atoms. Therefore, overlapping of electronic density between $\mathrm{H}$ atoms bound to $\mathrm{C}$ atoms and neighboring $\mathrm{C}$ atoms takes place. The strong covalent bonding between $\mathrm{C}$ and $\mathrm{H}$ atoms is confirmed also by results of thermal treatment of H-SWNTs. The H-SWNT C $1 s$ spectrum measured after annealing at the temperature $\left(T_{D H}\right)$ equal to hydrogenation temperature of the pristine SWNTs $\left(T_{H} \sim 530^{\circ} \mathrm{C}\right)$ demonstrated incompleteness of the H-SWNTs dehydrogenation (Fig. 4(b)).
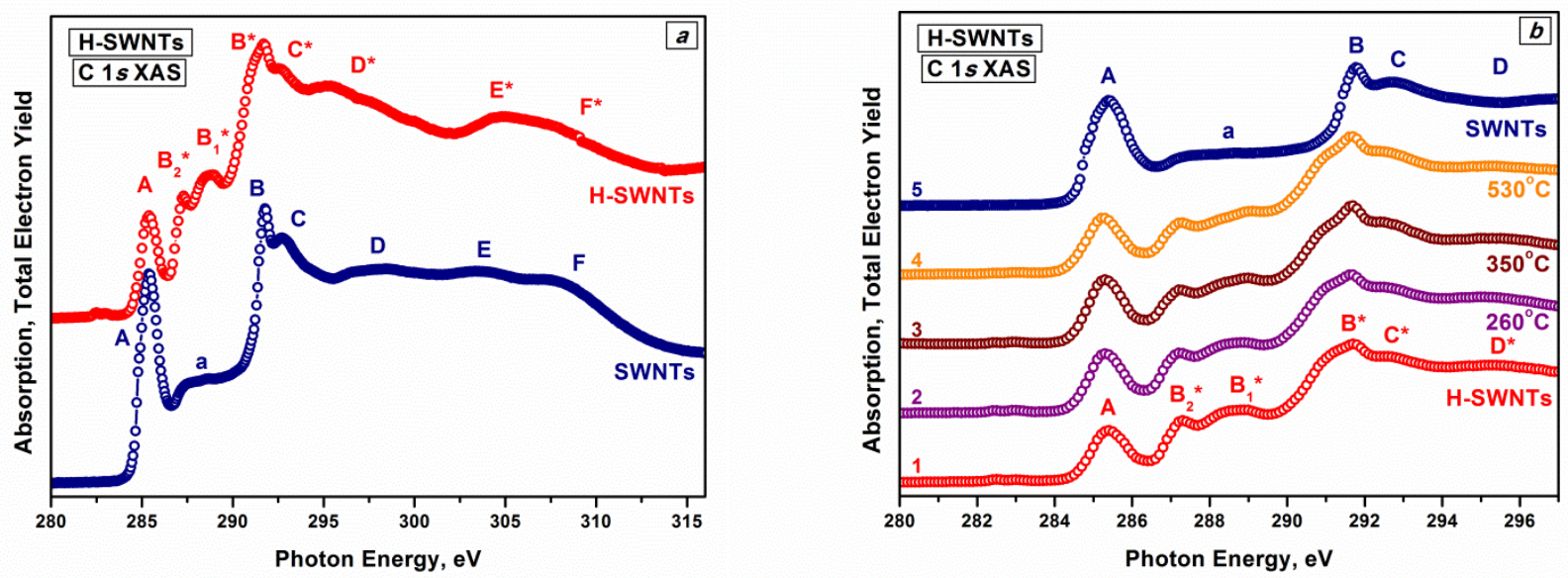

Fig. 4. The C $1 s$ X-ray absorption spectra of SWNTs and H-SWNTs before and after annealing. 
The investigation of H-SWNTs dehydrogenation can make it possible to estimate the type and power of carbon-hydrogen bonding and to find out whether the graphene mesh is distorted. Annealing is one of the possible methods of H-SWNTs dehydrogenation. The thermal annealing was performed under ultra-high vacuum conditions and started at the temperatures much lower than the hydrogenation one $\left(T_{H}\right)$. After the first two annealing stages at $T_{D H}=260^{\circ} \mathrm{C}$ and $310^{\circ} \mathrm{C}$ $(t=10$ minutes each), no changes were revealed in the $\mathrm{C} 1 \mathrm{~s}$ absorption spectra (Fig. 4(b), curve 2). Such results testify to the absence of changes in the H-SWNTs atomic structure in the probed region. Changes in the $\mathrm{C} 1 s$ absorption spectrum structure were observed for the first time only after the fourth annealing carried out during the same 10 minutes but at the temperature close to $T_{H}$ of pristine SWNTs $\left(T_{D H}=350^{\circ} \mathrm{C}\right)$. The changes manifested themselves in small reduction of bands $B_{1} *$ and $B_{2} *$ relative intensities and growth of bands $A$ and $B$ intensities (Fig. 4(b), curve 3). Bands $B_{1}^{*}$ and $B_{2} *$ in the $\mathrm{C} 1 s$ absorption spectrum correspond to the transitions of $\mathrm{C} 1 s$ electrons to the free hybridized states formed from $\mathrm{C} 2 p$ - and $\mathrm{H} 1 s$-states. Bands $A$ and $B$ correspond to the spectra of the initial regions of the hexagonal graphene mesh without any hydrogen atoms attached. These findings become even more evident after annealing at $T_{D H}=T_{H}$ $=500^{\circ} \mathrm{C}$ (not shown here). Obviously, such changes in the $\mathrm{C} 1 s$ absorption spectra of $\mathrm{H}-\mathrm{SWNTs}$ demonstrate the onset of the dehydrogenation process, i.e., partial breakage of C-H bonds. At the same time, next annealings performed at $T_{D H}=530^{\circ} \mathrm{C}(t=10-40$ minutes $)$ did not made the dehydrogenation more evident (Fig. 4(b), curve 4). Therefore, the experiment performed makes it possible to affirm that annealing of H-SWNTs does not lead to their complete dehydrogenation.

Table 1. Characteristics of the considered SWNT and H-SWNT models.

\begin{tabular}{cccccc}
\hline Model & $\mathrm{N}_{\mathrm{C}}$, at. & $\mathrm{N}_{\mathrm{H}, \text { at. }}$ & $\mathrm{E}_{\text {total }}, \mathrm{eV}$ & $\mathrm{E}_{\mathrm{coh}, \mathrm{eV}}$ & $\begin{array}{c}\Delta \mathrm{E}_{\text {total }} \\
\mathrm{eV} / \mathrm{H} \text {-atom }\end{array}$ \\
\hline Isolated C atom & 1 & - & -146.36 & - & - \\
\hline Isolated H atom & - & 1 & -11.93 & - & - \\
\hline SWNT (10,10) & 40 & - & -6306.70 & 452.20 & - \\
\hline $\begin{array}{c}\text { H-SWNT (10,10) } \\
\text { «2-outside» }\end{array}$ & 40 & 2 & -6337.63 & 459.27 & -3.53 \\
\hline $\begin{array}{c}\text { H-SWNT(10,10) } \\
\text { «outside-inside» }\end{array}$ & 40 & 2 & -6337.05 & 458.69 & -3.24 \\
\hline
\end{tabular}


Our experimental results were confirmed theoretically. First a non-hydrated $(10,10)$ nanotube was modeled, and its structural parameters, total energy and sublimation energy were determined by the DFT-LDA method (see Table 1 ). The nanotube $(10,10)$ unit cell contains 40 atoms, hence, attachment of a pair of hydrogen atoms provides the hydrogen concentration of 5 at.\%. The following cases of the atom attachment in pairs were considered: 1) outside (zig-zag orientation, Fig. 5(a)); 2) outside (armchair orientation); 3) one atom outside, the other inside (zig-zag orientation, Fig. 5(c)); 4) one atom outside, the other inside (armchair orientation, Fig. 5(b)); 5) inside.

Attachment of hydrogen atoms to nanotubes should occur in pairs because covalent bonds are formed by pairs of electrons, and, thus, the bond between the carbon and hydrogen atoms may be formed only if one of the nanotube $\pi$-covalent carbon-carbon bonds is broken. One of the electrons will be used to form a bond with the hydrogen atom, while the other becomes free after the $\mathrm{C}-\mathrm{C}$ bond breakage and remains non-compensated. This electron may be involved in attaching one more hydrogen atom. Therefore, hydrogen atoms are expected to attach to the nanotube surfaces and/or graphene layers in pairs.

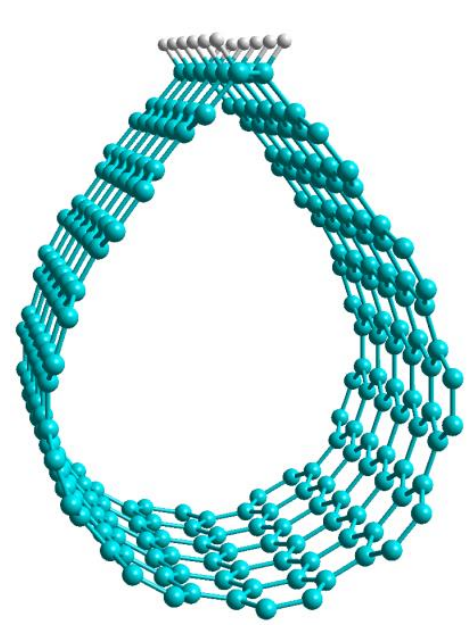

$\mathbf{a}$

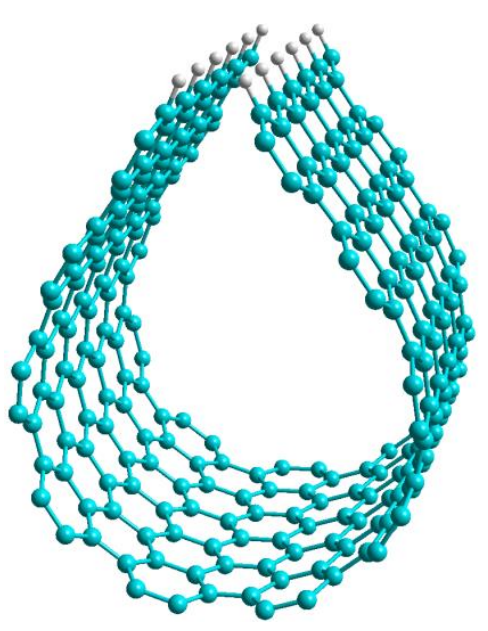

b

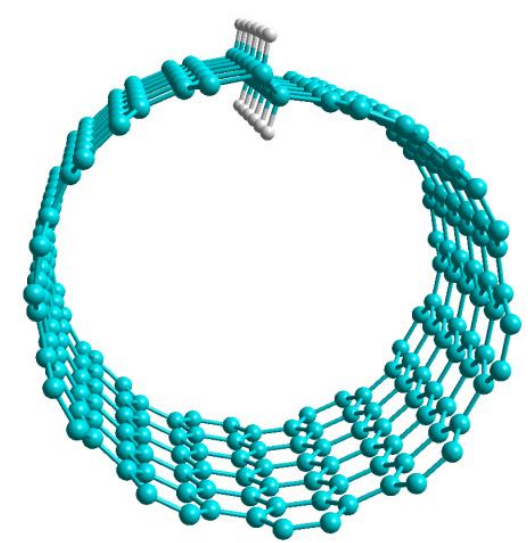

c

Fig. 5. Optimized structure of (10,10) SWNT with chemically adsorbed hydrogen atoms: from the outside in zig-zag (a) and armchair orientations (b), and from the outside and inside in zigzag orientation (c). The SWNT fragments presented in the figure contain six unit cells each.

The calculation results are presented in Table 1. The main characteristic based on which it is possible to estimate the energetic favorability of any given structural configuration is the specific incremental energy per hydrogen atom. This energy was calculated as a difference between total energies of hydrogenated single-wall carbon nanotubes and pristine ones (free of hydrogen), from which the total energy of two separate hydrogen atoms was then subtracted. 
The obtained value was divided in two. Formation of chemical bonds between the $\mathrm{H}$ and $\mathrm{C}$ atoms of a carbon nanotube results in significant deformation of the nanotube cylindrical shape.

The calculations showed that the most favorable version is attachment of a pair of hydrogen atoms in the zig-zag orientation outside the nanotube $(\Delta \mathrm{E}=-3.53 \mathrm{eV} / \mathrm{H}$-atom, Fig. $5(\mathrm{a})$ ), which is in good agreement with the results of other authors and proved the correctness of our calculations $[14,17,43]$. At the same time we should note that the hydrogen-SWNTs bonding energy calculated in this work (-3.53 to $-3.24 \mathrm{eV} / \mathrm{H}$-atom) is lower than the same bonding energy (-3.04 to $-1.68 \mathrm{eV} / \mathrm{H}$-atom) calculated in $[44,45]$. This difference is caused by the fact that in this work weak SWNTs hydrogenation was studied (with the relative content of adsorbed hydrogen of $\sim 5 \%$ ), while in works [44, 45] CNTs were completely hydrogenated. Thus, bonding of isolated hydrogen atoms chemically adsorbed on the SWNT surface should be stronger than that in completely hydrogenated SWNT. Hydrogen attachment outside the nanotube in the armchair orientation results in the nanotube destruction in the course of geometrical optimization (Fig. 5(b)). Structural configurations "one atom outside, the other inside" are less favorable than the first configuration (Table 1, Fig. 5(c)). Cases 2 and 3 of attaching hydrogen atoms to the CNT walls give one and the same result, namely, transformation of the nanotubes into graphene belts [46]. Thus, the most energetically favorable case is attaching hydrogen to the nanotube outside.

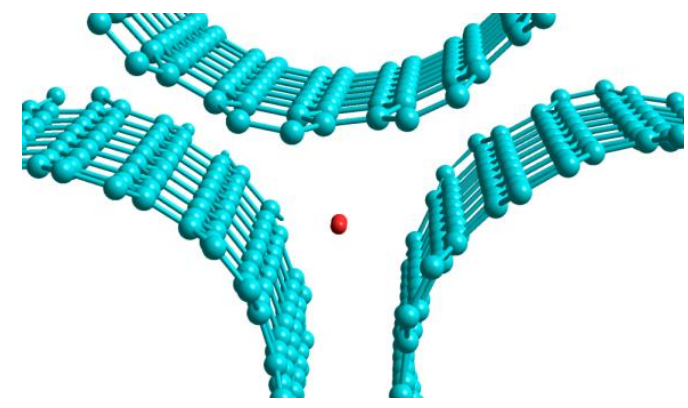

Fig. 6. Physically adsorbed $\mathrm{H}_{2}$ molecule in the $(10,10)$ SWNT bundles.

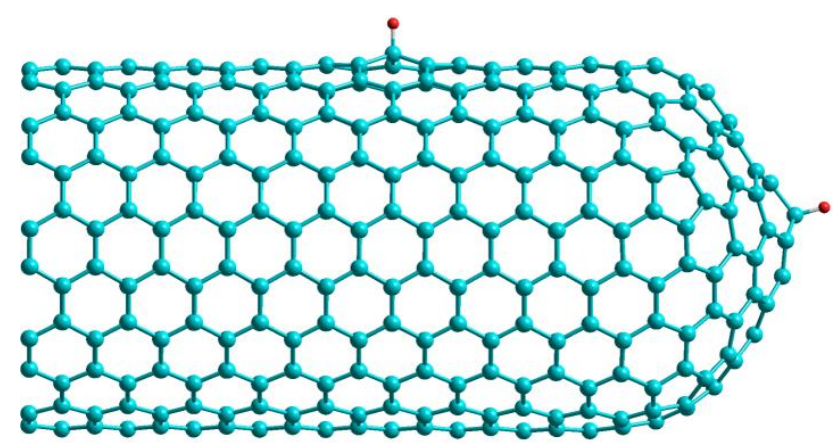

Fig. 7. Physically adsorbed $\mathrm{H}$ atoms on the wall and cups of the $(10,10)$ SWNTs.

The possibility of a hydrogen molecule penetration into the nanotube bundles was estimated by the molecular mechanics (MM2) method. Hydrogen can penetrate into the triangular intertube channels (Fig. 6). Location of hydrogen molecules inside the channels is energetically more favorable than outside the channels. This is nothing more than physical absorption due to van-der-Waals interactions, which was also assumed in [47]. After that, covalent chemical bonds between the hydrogen atoms and nanotube walls may arise. However, 
not the entire surface of the nanotubes located inside the bundle is accessible for hydrogenation but only the segments adjacent to the triangular pores.

Comparative estimation of bonding energies between hydrogen and nanotube walls and hydrogen and fullerene halves closing the nanotube ends showed that the bonding energies are higher at the nanotube ends where the curvature of the graphene layer forming the nanotube surface is maximal (Fig. 7).

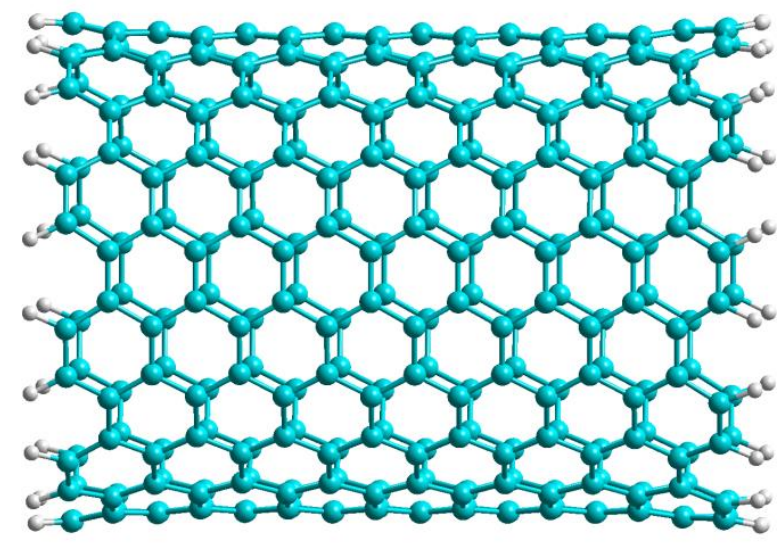

a

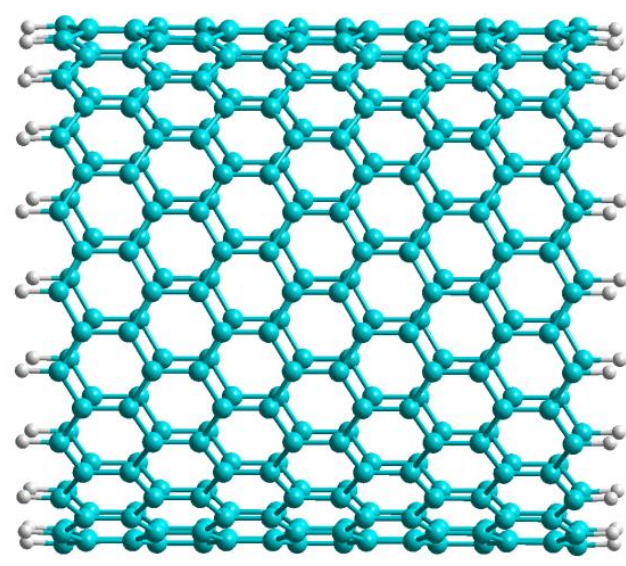

b

Fig. 8. The $\mathrm{C}_{320} \mathrm{H}_{40}$ fragment of the $(10,10)$ and $(20,0) \mathrm{H}-\mathrm{SWNT}$.

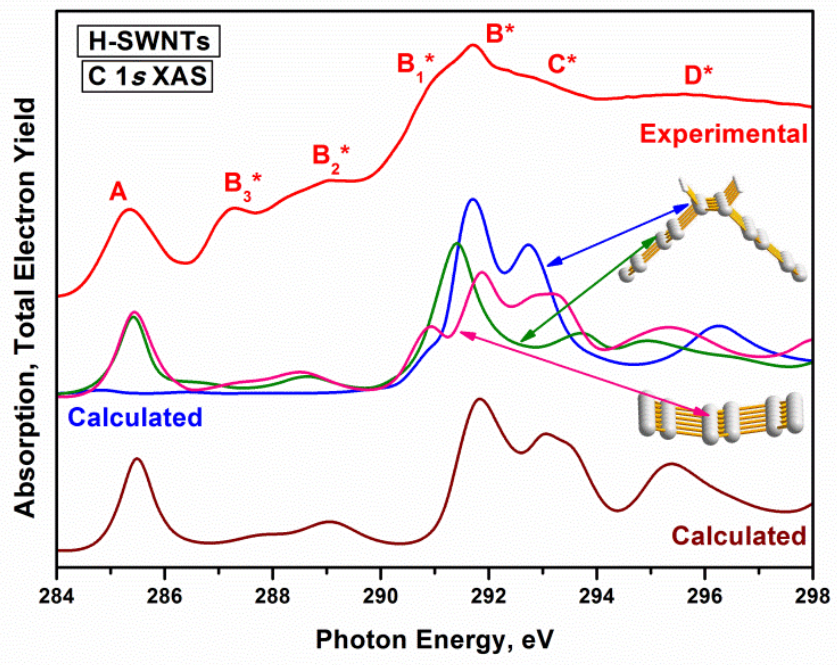

Fig. 9. The experimental and calculated C $1 s$ X-ray absorption spectra of H-SWNT.

The nanotube fragments of the "armchair" $(10,10)$ and "zig-zag" $(20,0)$ types were calculated (Fig. 8(a) and Fig. 8(b), respectively). Ends of those nanotubes remained open, while the dangling carbon-carbon bonds at the ends were compensated by the hydrogen atoms. The number of carbon and hydrogen atoms in the tubes' fragments was the same (320 carbon atoms 
and 40 hydrogen atoms). The nanotube diameters appeared to be equal (13.77 and $15.26 \AA$ ), which meant that the surface curvatures differed insignificantly. The optimization was performed by the molecular mechanics method (MM2). The energies were calculated by the semiempirical quantum-mechanical method PM3 [48]. The total bonding energy of the $(10,10)$ nanotube proved to be $-2401.43 \mathrm{eV}$ while that of the $(20,0)$ nanotube was $-2803.77 \mathrm{eV}$. The difference in these energies was caused by the difference in modes of attaching 40 hydrogen atoms to the fragment ends. This makes clear that the bonding energy between hydrogen and nanotube zig-zag ends is more favorable than that between hydrogen and nanotube armchair ends.

The simulation results were used to theoretically analyze the experimental C $K$-spectra. In our previous works, theoretical analysis of the experimental X-ray absorption spectra was already successfully used for simulation of the nanocompounds [49]. The theoretical CK-spectra were calculated based on the optimized structure of the $(10,10)$ SWNT with chemically adsorbed hydrogen atoms: namely, outside in the zig-zag configuration for which the minimal energies were obtained (Fig.5(a)). It was revealed earlier [40] that the theoretical C $K$-spectra in HSWNTs are highly sensitive to the choice of the absorbing atom the calculations are performed for. To ensure compliance with the experimental C $1 s$ NEXAFS spectrum of H-SWNTs that is an averaged signal from absorbing carbon atoms in different neighborhoods, the theoretical spectra should be calculated taking into account all the absorbing carbon atoms, both hydrogenbonded and non-hydrogen-bonded. In addition, as Fig. 5(a) shows, attachment of hydrogen atoms induces significant deformation of H-SWNTs relative to ideal SWNTs, which results in considerable changes in the local neighborhood of carbon atoms depending on their location. To take into account all these factors, three types of absorbing atoms were used in calculating $\mathrm{C} 1 \mathrm{~s}$ NEXAFS spectra, namely, a hydrogen-bonded carbon atom, a similar non-hydrogen-bonded carbon atom from the zig-zag chain neighboring to the hydrogenated chain, and non-hydrogenbonded carbon atom that is in the position diametrically opposite to the hydrogen-bonded carbon atom. Figure 9 illustrates the comparison of theoretical $\mathrm{C} K$-spectra calculated for absorbing carbon atoms with different local neighborhoods with the experimental H-SWNTs spectrum. Figure 9 demonstrates that the spectrum corresponding to the hydrogen-bonded carbon atom (blue curve) perfectly reproduces features $B, C$ and $D$ of the experimental spectrum. At the same time, this spectrum exhibits absolute absence of feature $A$ related to the $\pi$ resonance and of all the features between resonances $A$ and $B-C$, though earlier feature $B_{3}$ related to hydrogen, as well as low-intense feature $A$, was properly reproduced in the carbon atom spectra. In this model (Fig. 9), this might be caused by significant deformation of the graphene plane in the region of hydrogen atoms attachment and by almost absolute absence of electronic density overlapping 
between carbon atoms both bound chemically hydrogen atoms and carbon atoms neighboring to them. This contradicts to previous results obtained for the cases of insignificant graphene plane distortion. The second spectrum was calculated for a similar carbon atom from the chain neighboring to the hydrogenated chain (green curve). The spectrum reveals appearance of intense feature $A$ related to free $\pi$ states that are not occupied by hydrogen atoms in this case as well as appearance of feature $B_{2}$. Taking into account the hydrogenation degree, we can say that the main contribution to the experimental C $1 s$ NEXAFS spectra of H-SWNTs comes from nonhydrogen-bonded carbon atoms. Thus the third spectrum was calculated for the case when the atom on the opposite side of SWNT was chosen as the absorbing atom (pink curve). The features' shapes and positions in this spectrum are similar to those in the $C K$-spectrum calculated for ideal SWNT, which seems reasonable since the local neighborhood of the chosen absorbing atom is almost absolutely identical to the ideal SWNT neighborhood. The only difference is feature $B_{1}$ that is observed in the H-SWNT experimental spectrum and is absent in both the experimental and theoretical SWNT spectra. We suppose that this is caused by a stronger deformation of the graphene plane in the H-SWNT model as compared with the SWNT model. Thus we can note that consideration of partial contributions of spectra calculated for absorbing atoms with different local neighborhoods results in reproducing all the basic features of the experimental C $K$-spectrum. However, it should be noted that neither of the spectra reproduces intensities of features lying between the $A$ and $B$ resonances, which may be an evidence for overestimation of the nanotube deformation in simulation and confirm that inter-resonance features arise due to overlapping of electronic densities of hydrogen atoms and neighboring nonhydrogen-bonded carbon atoms.

\section{Conclusions}

In this work, we attempted to elucidate the CNT structure changes caused by hydrogenation using high-resolution near edge X-ray absorption fine structure (NEXAFS) spectroscopy and X-ray photoelectron spectroscopy (XPS). In parallel, the physical adsorption of hydrogen by SWNTs bundles was simulated using the molecular mechanics (MM2) and DFTLDA methods and applied to interpret the results of the experimental study. The CNTs models obtained as a result of such a simulation were used to theoretically analyze the absorption spectra. As a result, new aspects of the nature of interaction between hydrogen atoms and carbon atoms of single-wall carbon nanotubes were revealed, as well as preferable ways for SWNTs hydrogenation. It was found out that the hydrogen atoms should be attached to the nanotubes in twos. Hydrogen was shown to be able to penetrate into the triangular intertube channels. Due to van-der-Waals interactions, location of hydrogen molecules inside the channels is energetically 
more favorable than that outside the channels. Thus, chemical covalent bonds between hydrogen atoms and nanotube walls arise with formation of $\mathrm{C}_{4} \mathrm{H}$ and $\mathrm{C}_{2} \mathrm{H}$ phases. However, not the entire surface of the SWNTs located inside the bundles is accessible for hydrogenation but only the segments adjacent to the triangular pores.

\section{Acknowledgements}

This work was performed within the framework of the "Russian-German Laboratory at BESSY" bilateral Program. We thank HZB for the allocation of synchrotron radiation beamtime. G.Y. thanks the support from the Southern Federal University (Russia) through grant VnGr07/2017-30.

\section{References}

[1] G.A. Mesyats, M.D. Prokhorov, Hydrogen energetics and fuel cells, Herald of the Russian Academy of Sciences 74 (2004) 377-386.

[2] J.E. Fischer, Storing energy in carbon nanotubes, Chem. Innovation 30 (2000) 21-27.

[3] A.C. Dillon, K.M. Jones, T.A. Bekkedahl, C.H. Kiang, D.S. Bethune, M.J. Heben, Storage of hydrogen in single-walled carbon nanotubes, Nature 386 (1997) 377-379. https://doi.org/10.1038/386377a0.

[4] A.C. Dillon, M.J. Heben, Hydrogen storage using carbon adsorbents: past, present and future, Appl. Phys. A 72 (2001) 133-142. https://doi.org/10.1007/s003390100788.

[5] K. Murata, K. Kaneko, H. Kanoh, D. Kasuya, K. Takahashi, F. Kokai, M. Yudasaka, S. Iijima, Adsorption mechanism of supercritical hydrogen in internal and interstitial nanospaces of single-wall carbon nanohorn assembly, J. Phys. Chem. B 106 (2002) 11132-11138. https://pubs.acs.org/doi/pdf/10.1021/jp020583u.

[6] M-W. Zhao, Y-Y. Xia, Y-C. Ma, M-J. Ying, X-D. Liu, L-M. Mei, Tunable adsorption and desorption of hydrogen atoms on single-walled carbon nanotubes, Chin. Phys. Lett. 19 (2002) 1498-1500. doi: cpl.iphy.ac.cn/Y2002/V19/I10/014.

[7] A. Okati, A. Zolfaghari, F.S. Hashemi, N. Anousheh, H. Jooya, Hydrogen physisorption on Stone-Wales defect-embedded single-walled carbon nanotubes, Fuller. Nanotub. Car. N. 17 (2009) 324-335. doi: 10.1080/15363830902776599.

[8] A.R. Muniz, M. Meyyappan, D. Maroudas, Effects of hydrogen chemisorption on the structure and deformation of single-walled carbon nanotubes, Appl. Phys. Lett. 95 (2009) 163111-1-163111-3. doi: 10.1063/1.3095923. 
[9] M. Rzepka, P. Lamp, M.A. de la Casa-Lillo, Physisorption of hydrogen on microporous carbon and carbon nanotubes, J. Phys. Chem. B 102 (1998) 10894-10898. doi:10.1021/jp9829602.

[10] S.M. Lee, K.H. An, Y.H. Lee, G. Seifert, T. Frauenheim, A hydrogen storage mechanism in single-walled carbon nanotubes. J. Am. Chem. Soc. 123 (2001) 5059-5063. doi: $10.1021 / \mathrm{ja} 003751+$.

[11] F.L. Darkrim, D. Levesque, Monte Carlo simulations of hydrogen adsorption in singlewalled carbon nanotubes, J. Chem. Phys. 109 (1998) 4981-4984. doi: 10.1063/1.477109.

[12] J.R. Cheng, X.H. Yuan, L. Zhao, D.C. Huang, M. Zhao, L. Dai, R. Ding, GCMC simulation of hydrogen physisorption on carbon nanotubes and nanotube arrays, Carbon 42 (2004) 20192024. doi: 10.1016/j.carbon.2004.04.006.

[13] D. Silambarasan, V. Jayaraman Surya, V. Vasu, K. Iyakutti, Reversible and reproducible hydrogen storage in single-walled carbon nanotubes functionalized with borane, in: M.M. Rahman (Ed.), Carbon nanotubes - recent progress, IntechOpen, 2018, pp. 331-348. http://dx.doi.org/10.5772/intechopen.75763.

[14] J.S. Arellano, L.M. Molina, A. Rubio, M.J. Lopez, J.A. Alonso, Interaction of molecular and atomic hydrogen with $(5,5)$ and $(6,6)$ single-wall carbon nanotubes, J. Chem. Phys. 117 (2002) 2281-2288. https://doi.org/10.1063/1.1488595.

[15] S.S. Han, M.H. Lee, Adsorption properties of hydrogen on (10, 0) single-walled carbon nanotube through density functional theory, Carbon 42 (2004) 2169-2177. https://doi.org/10.1016/j.carbon.2004.04.025.

[16] D. Henwood, J. David Carey, Ab initio investigation of molecular hydrogen physisorption on graphene and carbon nanotubes, Phys. Rev. B 75 (2007) 245413. https://doi.org/10.1103/PhysRevB.75.245413.

[17] G.P. Miller, J. Kintigh, E. Kim, P.F. Weck, S. Berber, D. Tomanek, Hydrogenation of single-wall carbon nanotubes using polyamine reagents, J. Am. Chem. Soc. 130 (2008) 22962303. doi: 10.1021/jacs.6b11021.

[18] A.V. Talyzin, S. Luzan, I.V. Anoshkin, A.G. Nasibulin, H. Jiang, E.I. Kauppinen, V.M. Mikoushkin, V.V. Shnitov, D.E. Marchenko, D. Noréus, Hydrogenation, purification and unzipping of carbon nanotubes by reaction with molecular hydrogen: road to graphane nanoribbons, ACS NANO 5 (2011) 5132-5140. https://pubs.acs.org/doi/pdf/10.1021/nn201224k. [19] S. Pekker, J-P. Salvetat, E. Jakab, J-M. Bonard, L. Forro, Hydrogenation of carbon nanotubes and graphite in liquid ammonia, J. Phys. Chem. B 105 (2001) 7938-7943. doi: 10.1021/jp010642o. 
[20] M. Quintana, H. Traboulsi, A. Llanes-Pallas, R. Marega, D. Bonifazi, M. Prato, Multiple hydrogen bond interactions in the processing of functionalized multi-walled carbon nanotubes, ACS NANO 6 (2012) 23-31. doi: 10.1021/nn203471t.

[21] A. Nikitin, H. Ogasawara, D. Mann, R. Denecke, Z. Zhang, H. Dai, K. Cho, A. Nilsson, Hydrogenation of single-walled carbon nanotubes, Phys. Rev. Lett. 95 (2005) 225507. https://doi.org/10.1103/PhysRevLett.95.225507.

[22] P. Ruffieux, O. Gröning, M. Bielmann, P. Mauron, L. Schlapbach, P. Gröning, Hydrogen adsorption on $s p^{2}$-bonded carbon: Influence of the local curvature, Phys. Rev. B 66 (2002) 245416. doi: 10.1103/PhysRevB.66.245416.

[23] K. Nemeth, A. Pekker, F. Borondics, E. Jakab, N.M. Nemes, K. Kamaras, S. Pekker, Investigation of hydrogenated HiPCo nanotubes by infrared spectroscopy, Phys. Status Solidi B 247 (2010) 2855-2858. doi: 10.1002/pssb.201000329.

[24] E. Yoo, L. Gao, T. Komatsu, N. Yagai, K. Arai, T. Yamazaki, K. Matsuishi, T. Matsumoto, J. Nakamura, Atomic hydrogen storage in carbon nanotubes promoted by metal catalysts, J. Phys. Chem. B 108 (2004) 18903-18907. https://pubs.acs.org/doi/pdf/10.1021/jp047056q.

[25] M.M. Brzhezinskaya, V.E. Muradyan, N.A. Vinogradov, A.B. Preobrajenski, W. Gudat, A.S. Vinogradov, Electronic structure of fluorinated multi-walled carbon nanotubes, Phys. Rev. B 79 (2009) 155439. doi: 10.1103/PhysRevB.79.155439.

[26] A.V. Krestinin, A.V. Raevskii, N.A. Kiselev, G.I. Zvereva, O.M. Zhigalina, O.I. Kolesova, Optical activity effect in crystalline structures of purified single-wall carbon nanotubes, Chem. Phys. Lett. 381 (2003) 529. https://doi.org/10.1016/j.cplett.2003.10.020.

[27] K.P. Meletov, A.A. Maksimov, I.I. Tartakovskii, I.O. Bashkin, V.V. Shestakov, A.V. Krestinin, Yu.M. Shulga, K.S. Andrikopoulos, J. Arvanitidis, D. Christofilos, G.A. Kourouklis, Raman study of the high-pressure hydrogenated single-wall carbon nanotubes: In search of chemically bonded and adsorbed molecular hydrogen, Chem. Phys. Lett. 433 (2007) 335-339. https://doi.org/10.1016/j.cplett.2006.11.072.

[28] V.E. Antonov, I.O. Bashkin, S.S. Khasanov, A. Pmoravsky, Yu.G. Morozov, Yu.M. Shulga, Yu.A. Ossipyan, E.G. Ponyatovsky, Magnetic ordering in hydrofullerite $\mathrm{C}_{60} \mathrm{H}_{24}$, J. Alloys Compd. 330-332 (2002) 365-368. https://doi.org/10.1016/S0925-8388(01)01534-1.

[29] S.L. Molodtsov, S.I. Fedoseenko, D.V. Vyalikh, I.E. Iossifov, R. Follath, S.A. Gorovikov, M.M. Brzhezinskaya, Y.S. Dedkov, R. Püttner, J.-S. Schmidt, V.K. Adamchuk, W. Gudat, G. Kaindl, High-resolution Russian-German beamline at BESSY, Appl. Phys. A 94 (2009) 501505. https://doi.org/10.1007/s00339-008-4916-1.

[30] R. Hesse, M. Weiß, R. Szargan, P. Streubel, R. Denecke, Comparative study of the modelling of the spectral background of photoelectron spectra with the Shirley and improved 
Tougaard methods, J. Electron. Spectrosc. Relat. Phenom. 186 (2013) 44-53. doi: 10.1016/j.elspec.2013.01.020.

[31] N.L. Allinger, Conformational analysis. 130. MM2. A hydrocarbon force field utilizing V1 and V2 torsional terms, J. Am. Chem. Soc. 99 (1977) 8127-8134. doi: 10.1021/ja00467a001.

[32] P. Giannozzi, S. Baroni, N. Bonini, M. Calandra, R. Car, C. Cavazzoni, D. Ceresoli, G.L. Chiarotti, M. Cococcioni, I. Dabo, A. Dal Corso, S. Fabris, G. Fratesi, S. de Gironcoli, R. Gebauer, U. Gerstmann, C. Gougoussis, A. Kokalj, M. Lazzeri, L. Martin-Samos, N. Marzari, F. Mauri, R. Mazzarello, S. Paolini, A. Pasquarello, L. Paulatto, C. Sbraccia, S. Scandolo, G. Sclauzero, A. P. Seitsonen, A. Smogunov, P. Umari, R. M. Wentzcovitch. QUANTUM ESPRESSO: a modular and open-source software project for quantum simulations of materials, J. Phys: Condens. Matter. 21 (2009) 395502. doi: 10.1088/0953-8984/21/39/395502.

[33] J.P. Perdew, A. Zunger, Self-interaction correction to density-functional approximations for many-electron systems, Phys. Rev. B 23 (1981) 5048-5079. doi: 10.1103/PhysRevB.23.5048.

[34] Y. Joly, Calculating X-ray absorption near-edge structure at very low energy, J. Synch. Rad. 10 (2003) 58-63. doi: 10.1107/S0909049502017211.

[35] T. Susi, T. Pichler, P. Ayala, X-ray photoelectron spectroscopy of graphitic carbon nanomaterials doped with heteroatoms, Beilstein J. Nanotechnol. 6 (2015) 177-192. doi: 10.3762/bjnano.6.17.

[36] A.A. Eliseev, L.V. Yashina, M.M. Brzhezinskaya, M.V. Chernysheva, M.V. Kharlamova, N.I. Verbitsky, A.V. Lukashin, N.A. Kiselev, A.S. Kumskov, R.M. Zakalyuhin, J.L. Hutchison, B. Freitag, A.S. Vinogradov, Structure and electronic properties of AgX@SWNT (X=Cl, Br, I) intercalated single-walled carbon nanotubes, Carbon $48 \quad$ (2010) 2708-2721. doi:10.1016/j.carbon.2010.02.037.

[37] S. Huefner, Photoelectron spectroscopy, Springer, Berlin, 1996.

[38] M.M. Brzhezinskaya, E.M. Baitinger, Plasmons in carbon nanotubes, in: D.A. Martin (Ed.), Trends in carbon nanotube research, Nova Science, New York, 2005, pp. 235-275.

[39] D. Haberer, C.E. Giusca, Y. Wang, H. Sachdev, A.V. Fedorov, M. Farjam, S. A. Jafari, D.V. Vyalikh, D. Usachov, X. Liu, U. Treske, M. Grobosch, O. Vilkov, V.K. Adamchuk, S. Irle, S.R.P. Silva, M. Knupfer, B. Büchner, A. Grüneis, Evidence for a new two-dimensional $\mathrm{C}_{4} \mathrm{H}-$ type polymer based on hydrogenated graphene. Adv. Mater. 23 (2011) 4497-4503. https://doi.org/10.1002/adma.201102019.

[40] M. Brzhezinskaya, G. Yalovega, V. Shmatko, A. Krestinin, I. Bashkin, E. Bogoslavskaja, Electronic structure of hydrogenated carbon nanotubes studied by core level spectroscopy, J. Elect. Spect. Rel. Phen. 196 (2014) 99-103. doi: 0.1016/j.elspec.2013.12.013. 
[41] A. Fujimoto, Y. Yamada, M. Koinuma, S. Sato, Origins of $\mathrm{sp}^{3} \mathrm{C}$ peaks in $\mathrm{C} 1 \mathrm{~s} \mathrm{X}$-ray photoelectron spectra of carbon materials, Anal. Chem. 88 (2016) 6110-6114. doi:10.1021/acs.analchem.6b01327.

[42] J. Stöhr, NEXAFS spectroscopy, Springer-Verlag, Berlin, 1992.

[43] V. Meregalli, M. Parrinello. Review of theoretical calculations of hydrogen storage in carbon-based materials, Appl. Phys. A 72 (2001) 143-146. doi: 10.1007/s003390100789.

[44] R.M.A. Khalil, F. Hussain, M. Imran, A.M. Rana, G. Murtaza, ab initio study of the exohydrogenated single wall carbon nanotubes, Physica B: Condensed Matter 552 (2019) 124-129. doi: 10.1016/j.physb.2018.09.044.

[45] R.M.A. Khalil, F. Hussain, A.M. Rana, M. Imran, Thermodynamics and vibrational study of hydrogenated carbon nanotubes: a DFT study, Physica B: Condensed Matter 530 (2018) 307311. doi: 10.1016/j.physb.2017.11.084.

[46] D.V. Kosynkin, A.L. Higginbotham, A. Sinitskii, J.R. Lomeda, A. Dimiev, B. Katherine Price, J.M. Tour, Longitudinal unzipping of carbon nanotubes to form graphene nanoribbons, Nature 458 (2009) 872-877. doi:10.1038/nature07872.

[47] H. Cheng, A.C. Cooper, G.P. Pez, M.K. Kostov, P. Piotrowski, S.J. Stuart. Molecular dynamics simulations on the effects of diameter and chirality on hydrogen adsorption in single walled carbon nanotubes, J. Phys. Chem. B 109 (2005) 3780-3786. doi: 10.1021/jp045358m.

[48] J.J.P. Stewart, Optimization of parameters for semiempirical methods I. Method, J. Comput. Chem. 10 (1989) 209-220. doi: 10.1002/jcc.540100208.

[49] A.N. Kravtsova, G.E. Yalovega, A.V. Soldatov, W.S. Yan, S.Q. Wei, The atomic structure of $\mathrm{Fe}_{100-\mathrm{x}} \mathrm{Cu}_{\mathrm{x}}$ nanoalloys: X-ray absorption analysis, J. Alloys Compd. 469 (2009) 42-49. https://doi.org/10.1016/j.jallcom.2008.02.017. 